\title{
Connecting diffusion and entropy of bulk water at the single particle level $^{\dagger}$
}

\author{
DEBASIS SAHA and ARNAB MUKHERJEE* (D) \\ Indian Institute of Science Education and Research Pune, Pune, Maharashtra 411 008, India \\ E-mail: arnab.mukherjee@iiserpune.ac.in; dr.arnab.mukherjee@gmail.com
}

MS received 31 January 2017; revised 19 May 2017; accepted 1 June 2017

\begin{abstract}
The relation between the dynamic (e.g., diffusion) and thermodynamic (e.g., entropy) properties of water and water-like liquids has been an active area of research for a long time. Although several studies have investigated the diffusivity and entropy for different systems, these studies have probed either the configurational entropy or the excess entropy of the overall system. In this study, we focus on the entropy of water at a single molecule level at different temperatures. We have used a method developed in our group to calculate the translational and rotational entropy of individual water molecules at various temperatures. We find that the single water translational and rotational entropy exhibit a transition at around $240 \mathrm{~K}$. The translational entropy of individual water molecules shows a consistent variation with change in temperature whereas the variation in the case of rotational entropy is much smaller at different temperatures. We have also calculated diffusion coefficients of water molecules at these temperatures. We find that diffusion also shows the well-known fragile to strong crossover transition at around the same temperature where transition in entropy values has been seen. We have calculated both kinetic and thermodynamic fragilities and crossover points using diffusion and single water translational entropy values. Finally, we correlate the diffusion and translational entropy of individual water molecules using an analog of the Adam-Gibbs relation.
\end{abstract}

Keywords. Single water entropy; Diffusion coefficient; Fragility.

\section{Introduction}

The properties of a liquid can be characterised by its dynamical quantities such as diffusion coefficient and relaxation times, or by its thermodynamic and structural properties such as entropy and structure factor. At equilibrium, the dynamic and thermodynamic quantities must be related. For example, the diffusivity of a liquid gets modified on the basis of the thermodynamic condition of the system. At low temperatures, the rearrangement of molecules responsible for the diffusion is dominated by jumps across different local energy minima, ${ }^{1}$ and these energy minima for a liquid correspond to the different accessible configurations at the corresponding thermodynamic state. ${ }^{2}$ Thus, the configurational entropy and diffusivity for the molecules in liquid systems are connected. However, the translational diffusion of the molecules is likely to influence the translational entropy of the system and the variation of these two quantities can be correlated.

\footnotetext{
*For correspondence

${ }^{\dagger}$ Dedicated to the memory of the late Professor Charusita Chakravarty.
}

Over the years, different approaches have been adopted to connect the entropy with diffusion. A number of studies in this context have discussed the relation between diffusion and the excess entropy of the system, $\mathrm{S}_{\mathrm{ex}}$. The excess entropy denotes the difference between the actual entropy of the system and the system's entropy if it behaves as an ideal gas at the same condition. An empirical relation between diffusion and this excess entropy was given by Rosenfeld as, ${ }^{3,4}$

$$
\mathrm{D}=\mathrm{a} \exp \left(\frac{\mathrm{bS}}{\mathrm{k}_{\mathrm{B}}}\right)
$$

where $\mathrm{k}_{\mathrm{B}}$ is the Boltzmann constant and, a and $b$ are empirical constants. Several studies have used the excess entropy to connect the thermodynamics with the dynamics of the systems for different models of water and water-like liquids. ${ }^{5-14}$ Thermodynamic states of various liquid models that mimic real systems were varied to investigate the correlation between diffusion and entropy. The excess entropy used in these studies can be expanded into a $n$-body contributions. ${ }^{15} \mathrm{~S}_{\mathrm{ex}}$ has often been approximated to a two-body excess entropy $S_{2}$ that depends on the radial distribution function $\mathrm{g}(\mathrm{r})$ as, 
$\mathrm{S}_{2}=-2 \pi \rho \mathrm{k}_{\mathrm{B}} \int\{\mathrm{g}(\mathrm{r}) \ln [\mathrm{g}(\mathrm{r})]-[\mathrm{g}(\mathrm{r})-1]\} \mathrm{r}^{2} \mathrm{dr}$.

A universal relation between the $\mathrm{S}_{2}$ and diffusion coefficient was given by Dzugutov ${ }^{16}$ which was tested for several systems. ${ }^{16}$ Recently, Seki and Bagchi provided a statistical mechanical derivation of the Rosenfeld relation and showed that this equation remains valid for any dimension. ${ }^{17}$ The other popular approach to connect diffusion with entropy comes from the relation between the configurational entropy $S_{C}$ with the diffusion coefficient. The quantitative relation between these two was given by Adam and Gibbs (AG) in the following form. ${ }^{18}$

$$
\mathrm{D}^{-1}(\mathrm{~T})=\mathrm{D}^{-1}\left(\mathrm{~T}_{0}\right) \exp \left\{\mathrm{A}_{\mathrm{AG}} /\left(\mathrm{T} \mathrm{S}_{\mathrm{Conf}}\right)\right\},
$$

Where $\mathrm{D}\left(\mathrm{T}_{0}\right)$ is the diffusion at a higher temperature $\mathrm{T}_{0}$. $\mathrm{A}_{\mathrm{AG}}$ is a constant known as Adam-Gibbs parameter. In different studies, the $\mathrm{S}_{\mathrm{Conf}}$ has been calculated by subtracting the vibrational entropy from the total entropy as, $\mathrm{S}_{\text {Conf }}(\mathrm{T})=\mathrm{S}_{\text {Total }}(\mathrm{T})-\mathrm{S}_{\text {vib }}(\mathrm{T})$.

Thermodynamic integration has been widely used in determining the total entropy of the system with water and water-like liquids. ${ }^{19-22}$ The vibrational entropy is calculated by making harmonic approximation to the local potential energy minimum. The constant $\mathrm{A}_{\mathrm{AG}}$ in the AG relation has been associated with the high temperature activation energy, ${ }^{20}$ and it has been found to depend strongly on the density of the system. ${ }^{22,23}$ Most of the studies devoted to the relation between diffusion and entropy focused on the thermodynamic and dynamic properties of the whole system. However, an understanding of the behaviour of individual water molecules in terms of thermodynamics and dynamics is missing.

Despite the numerous studies, the behaviour of water at low temperatures remains an area of active research due to its uniqueness compared to other liquids. One such distinct property is the existence of a singular temperature close to which various thermodynamic properties such as compressibility and thermal expansion coefficient diverge. ${ }^{24}$ The study of the glass transition (GT) of water poses various challenges due to the dramatic slowdown of macroscopic properties such as viscosity or self-diffusion coefficient as well as the microscopic property such as translational correlation time. These changes ought to govern the thermodynamic properties of the water molecules. Hence, a correlation between the thermodynamic properties with the dynamics of the system can provide a general pattern to predict the thermodynamic behaviour of liquids from the knowledge of the dynamic quantities. The crystallization of water makes different experimental techniques inadequate to study the super-cooled region of liquid water. Hence, the dynamics of the super-cooled water has been measured by confining water in nano-sized pores. ${ }^{25-27}$ Computer simulations have been very effective in exploring both the dynamic and thermodynamic properties of super-cooled water.

In this study, we have used molecular dynamics (MD) simulation and have adopted a different approach to establish a connection between the thermodynamic and dynamic properties of individual water molecules. We have calculated the translational and rotational entropy of individual water molecules at various temperatures using a method developed recently in our group. ${ }^{28} \mathrm{Sin}-$ gle molecule translational entropy was also calculated by Siebert et al., ${ }^{29}$ where they used free volume to calculate the effect of translational entropy in binding processes. Our method, however, is based on the volume accessed by a single molecule during the dynamics. We have found a systematic change for translational entropy with temperature whereas the rotational entropy faces a sharp change at a particular temperature. The calculations of self-diffusion coefficient (D) shed light on this phenomenon where we find a transition in the dynamics at around the same temperature where a dip is observed in entropy values indicating a fragile-tostrong crossover (FSC) transition in water. Thus, from the entropy and diffusion data, we have discussed kinetic and thermodynamic fragilities ${ }^{30,31}$ and the associated dynamical temperatures for water. We have obtained the temperature at which the translational entropy contribution originating from the movement of individual water oxygen becomes zero. Finally, to relate the single water translational entropy with $\mathrm{D}$, we propose the generalization of the Adam-Gibbs relation which connects the configurational entropy with the translational relaxation time.

\section{Computational methods}

\subsection{System set-up and simulation details}

All-atom molecular dynamics simulations were carried out to obtain the entropy and diffusion coefficients of water molecules. We have used the TIP5P/E water model ${ }^{32}$ for this study which has been proven to exhibit correct density trend with the variation of temperature. Our system contains 1000 water molecules. The entropies have been calculated from simulations at temperatures 220, 230, 240, 250, 260, 273, 285, 300,315 , and $330 \mathrm{~K}$. Diffusion coefficients were calculated for two additional temperatures 200 and $210 \mathrm{~K}$ in addition to the above temperatures. Since the fragile-to-strong crossover was observed at $240 \mathrm{~K}$, obtaining converged value for entropy below this temperature becomes computationally expensive.

We have used GROMACS ${ }^{33}$ molecular dynamics program for carrying out the simulations. The bulk water system was 


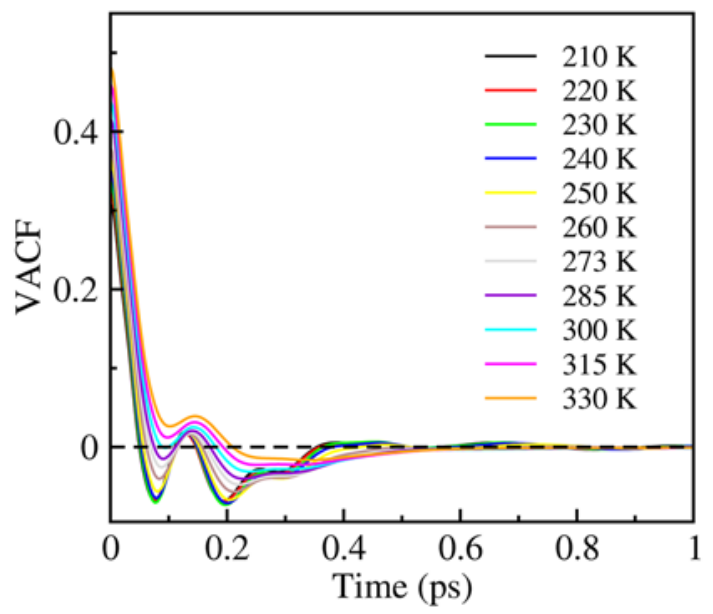

Figure 1. Time dependence of the velocity auto correlation function (VACF) of water at different temperatures.

first energy minimized using the steepest descent method, ${ }^{34}$ followed by heating up to the temperature required in each case using Berendsen thermostat ${ }^{35}$ with a coupling constant of $0.2 \mathrm{ps}$. Each system was equilibrated for $10 \mathrm{~ns}$ at constant temperature and pressure of 1 bar using Nosé-Hoover thermostat ${ }^{36,37}$ and Parrinello-Rahman barostat, ${ }^{38}$ respectively, where the coupling constants were kept at 0.2 ps for both. Long equilibration allowed the system to attain their natural density at atmospheric pressure. No constraint was applied in controlling the density of the systems. PME electrostatics ${ }^{39}$ were used for treating the electrostatic interactions. Cut-off distance of $10 \AA$ was used for both electrostatic and van der Waals interactions. The density pattern was then compared to the experimental values shown by Mallamace et al., ${ }^{40}$ to verify the accuracy of the simulations. The simulations were continued further for $200 \mathrm{~ns}$ for systems at temperatures 250 $\mathrm{K}$ and below to obtain converged entropy values under same NPT condition. For temperatures above this, only $100 \mathrm{~ns} \mathrm{sim-}$ ulations were enough to obtain converged entropy values. For calculating the diffusion coefficients, the equilibrated systems were subjected to short, $500 \mathrm{ps}$, simulations with the frames saved at every 4 fs to enable an accurate estimation of the velocity auto correlation function (VACF). Diffusion coefficients were calculated from VACF using the following Green-Kubo relation, ${ }^{41}$

$$
\mathrm{D}=\frac{1}{\mathrm{~d}} \int_{0}^{\infty} \mathrm{dt}\langle\overrightarrow{\mathrm{V}}(0) \cdot \overrightarrow{\mathrm{V}}(\mathrm{t})\rangle
$$

where $\mathrm{d}$ is the dimensionality and $\overrightarrow{\mathrm{V}}$ is the velocity vector. The VACF profiles for water at different temperatures are shown in Figure 1.

\subsection{Method for translational and rotational entropy for individual water}

Due to the diffusive nature of water molecules, standard method used for biomolecular entropy calculation ${ }^{42}$ cannot be used to estimate the entropy of water. Hence, in our calculations, the method known as permutation reduction (PR) ${ }^{43,44}$ developed by Grubmuller and co-workers have been used that considers the water to be indistinguishable and obtain an effect localized configuration space of a water molecule close to a particular region. More details can be found elsewhere. ${ }^{28}$ Each of the localized permuted water molecules was subjected to quasi-harmonic analyses to estimate the translational entropy individually. For that, we obtain the covariance matrix of translational fluctuation of the permuted water molecule along $\mathrm{X}, \mathrm{Y}$, and $\mathrm{Z}$ direction giving rise to a $3 \times 3$ matrix. Diagonalization of this matrix provides three eigenvalues $\left(\lambda_{i}, i=1,2,3\right)$ and consequently three frequencies $\omega_{\mathrm{i}}=\left(\mathrm{k}_{\mathrm{B}} \mathrm{T} / \lambda_{i}\right)^{1 / 2}, i=1,2,3$. With these frequencies, the translational entropy was calculated using the expression for entropy of solid state quantum harmonic oscillator shown in the following. ${ }^{45}$

$$
\mathrm{S}_{\mathrm{tr}}^{\mathrm{QH}}=\mathrm{k}_{\mathrm{B}} \sum_{\mathrm{i}=1}^{3} \frac{\hbar \omega_{\mathrm{i}} / \mathrm{k}_{\mathrm{B}} \mathrm{T}}{\mathrm{e}^{\hbar \omega_{\mathrm{i}} / \mathrm{k}_{\mathrm{B}} \mathrm{T}}-1}-\ln \left(1-\mathrm{e}^{-\hbar \omega_{\mathrm{i}} / \mathrm{k}_{\mathrm{B}} \mathrm{T}}\right),
$$

where, $h=\frac{h}{2 \pi}$, $h$ is the Planck's constant, T denotes the temperature, and $\mathrm{k}_{\mathrm{B}}$ is the Boltzmann constant. Considering the classical limit at high temperature $\left(\hbar \omega_{\mathrm{i}} / \mathrm{k}_{\mathrm{B}} \mathrm{T}<1\right)$, the above formula can be written in the following form.

$$
\mathrm{S}_{\mathrm{tr}}^{\mathrm{QH}}=\mathrm{k}_{\mathrm{B}} \sum_{\mathrm{i}=1}^{3} 1-\ln \left(1-\hbar \omega_{\mathrm{i}} / \mathrm{k}_{\mathrm{B}} \mathrm{T}\right)=\mathrm{C}(\mathrm{T})+\mathrm{k}_{\mathrm{B}} \ln \mathrm{V},
$$

where, $\mathrm{C}=\left(3-\ln \left(\frac{4 \pi}{3}\right)-\frac{3}{2} \ln \left(\frac{\mathrm{h}^{2}}{\mathrm{~K}_{\mathrm{B}} \mathrm{T}}\right)\right.$ and $\mathrm{V}=4 \pi / 3\left(\frac{1}{2 \sqrt{2}}\right)$ $\left(\lambda_{1} \lambda_{2} \lambda_{3}\right)^{\frac{1}{2}}$. Here, $\left(\lambda_{i}\right)^{1 / 2}, i=1,2,3$ represents the length of the principal axes of the ellipsoidal volume that a permuted water molecule occupies. The permuted water molecules were also used for calculating the rotational entropy of individual water molecules, as mentioned in detail elsewhere. ${ }^{28}$ We have shown recently that the calculated entropy values correlate well (correlation coefficient of 0.97) with the reported experimental values of entropy in the solvation shell of ions. ${ }^{46}$ The good correlation found between the calculated and experimental entropy established the reliability of our method. Since we discuss the entropy of individual water molecules for bulk water system, the permutation of water molecules has been done by keeping one water molecule at the center of the box followed by ordering other water molecules around the central one. Since the central water molecule remains static in calculation, the entropy of water molecules immediately next to it does not represent the bulk water; rather, it represents the first solvation shell water around another water molecule. Hence, we consider the entropy of water molecules away from the central water molecule. The average entropy values of 40 such water molecules were considered as the single water entropy at that particular temperature. The convergence of translational entropy values for a few water molecules are shown in Figure 2 for some temperatures. Similar convergence was found for rotational entropy as well (figure not shown). 


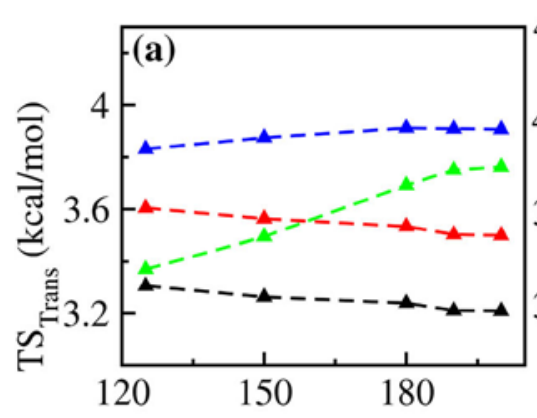

120
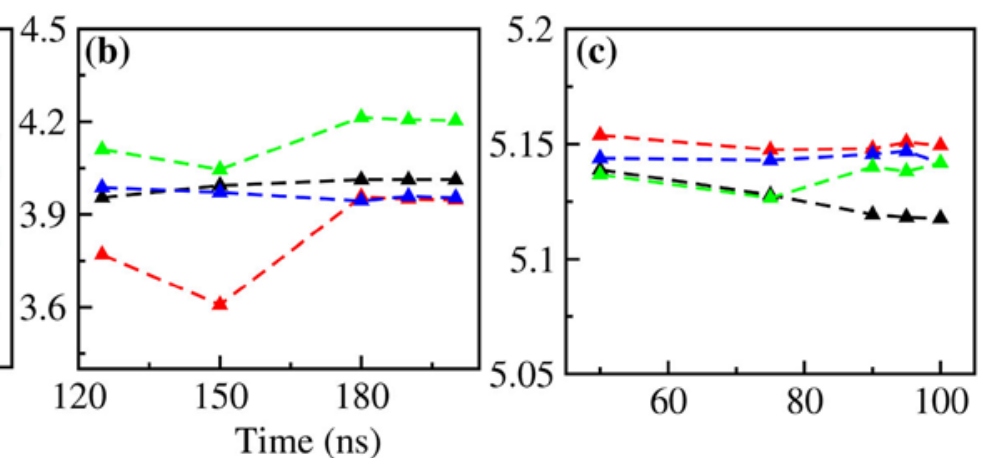

Figure 2. The convergence of translational entropy values for simulation at (a) $220 \mathrm{~K}$, (b) $230 \mathrm{~K}$, and (c) $273 \mathrm{~K}$ for four different water molecules. The black, red, blue, and green colours indicate the $40^{\text {th }}, 50^{\text {th }}$, $60^{\text {th }}$, and $70^{\text {th }}$ water molecule with respect to distance from the central water molecule.

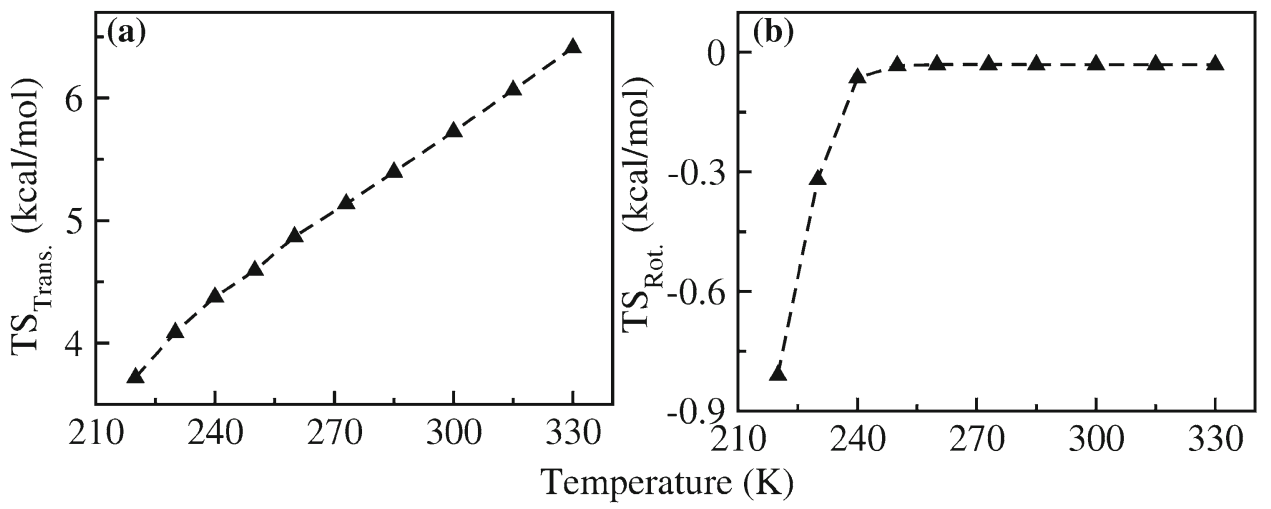

Figure 3. (a) The translational entropy and (b) the rotational entropy of individual water molecules with respect to temperature. The dashed lines here are only to guide the eyes.

\subsection{Fragility}

Here, we discuss a quantity called fragility, ${ }^{30,31}$ which quantifies the rate at which any dynamic quantity such as relaxation time, inverse diffusivity, or viscosity grows with temperature. In this study, the kinetic fragility is obtained by studying the temperature dependence of the dynamic quantity, diffusion coefficients above $250 \mathrm{~K}$ only due to the divergence of the diffusivity at the low temperature region. We plot the inverse of the diffusion coefficient against temperature using the VogelFulcher-Tammann (VFT) equation of the following form: ${ }^{19}$

$$
\mathrm{D}^{-1}(\mathrm{~T})=\mathrm{D}_{0}^{-1} \exp \left[\frac{1}{\mathrm{~K}_{\mathrm{VFT}}\left(\frac{\mathrm{T}}{\mathrm{T}_{\mathrm{VFT}}}-1\right)}\right],
$$

where, $\mathrm{K}_{\mathrm{VFT}}$ is the kinetic fragility marker and $\mathrm{T}_{\mathrm{VFT}}$ is the temperature at which the diffusivity diverges. $\mathrm{D}_{0}$ is the high temperature diffusivity.

In general, the thermodynamic fragility is studied by evaluating the temperature dependence of configurational entropy. The temperature dependence of $\mathrm{S}_{\mathrm{Conf}}$ is given as, ${ }^{22}$

$$
\mathrm{TS}_{\text {Conf. }}=\mathrm{K}_{\mathrm{T}}\left(\frac{\mathrm{T}}{\mathrm{T}_{\mathrm{K}}}-1\right)
$$

where, $\mathrm{K}_{\mathrm{T}}$ is the thermodynamic fragility marker and $\mathrm{T}_{\mathrm{K}}$ is the Kauzmann temperature. ${ }^{18}$ At $\mathrm{T}_{\mathrm{K}}$, the entropy contribution from configurations become zero and the entropy reaches the crystalline entropy. The AG relation (Eq. 3) connects between dynamics and thermodynamics, while Eq. 8 provides a thermodynamic relation and Eq. 7 gives a dynamic relation. Here, we study the thermodynamic fragility by using the translational entropy variation with temperature. The extrapolation of the values provides the temperature at which the contribution to translational entropy originating from the movements of water oxygen atoms becomes zero.

\section{Results and Discussion}

\subsection{Single water entropy variation with temperature}

In this section, we discuss the variation of entropy of individual water molecules at different temperatures. Hence, in Figure 3, we show the variation of $\mathrm{TS}_{\text {Trans }}$ and $\mathrm{TS}_{\text {Rot }}$ for individual water molecules at different temperatures. As can be seen from the figure, there is a deviation from linear behaviour in the translational entropy values at $240 \mathrm{~K}$. The deviation is more prominent in the 

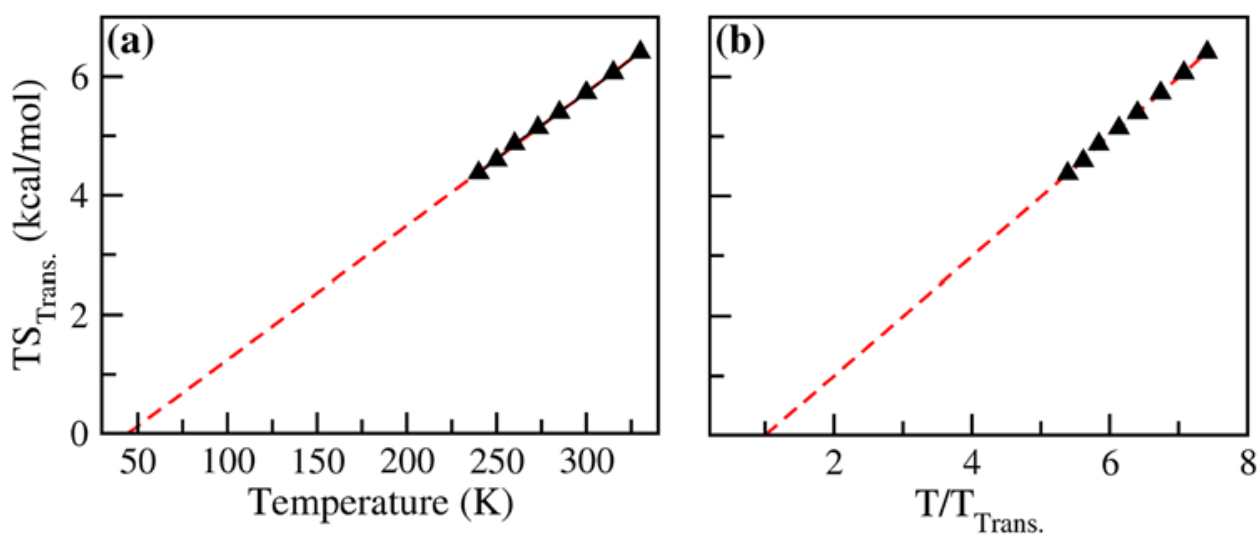

Figure 4. (a) Extrapolation of $\mathrm{TS}_{\text {Trans. values to the temperature where the translational }}$ entropy value reaches zero. This temperature is denoted as $\mathrm{T}_{\text {Trans. }}$ here. (b) The variation of $\mathrm{TS}_{\text {Trans. }}$ with $\mathrm{T} / \mathrm{T}_{\text {Trans. }}$ The slope of this line gives the analog of thermodynamic fragility value, $\mathrm{K}_{\text {Trans. }}$ which is found to be 0.02 from translational entropy values of individual water molecules.

case of rotational entropy. It is previously known that water undergoes a fragile-to-strong crossover transition at temperature close to $225 \mathrm{~K} .{ }^{47,48}$ This transition is clearly indicated in the entropy values as the system is expected to get stuck in local minima at that temperature. The time scale required to go from one energy basin to another will be much longer at these temperatures and could be inaccessible through molecular dynamics simulation. However, the molecules at these low temperatures will still have vibrations around their mean positions which will be expected to contribute to the translational entropy values in our calculations. Hence, the translational entropy observed at lower temperatures can be expected to be close to the crystalline entropy values. The values obtained here for translational entropies are found to be slightly higher than the reported absolute translational entropy values by Lin et al., ${ }^{49}$ using two-phase thermodynamic model (2PT) method for several water models. However, the water model and also the approach used here is very different from the $2 \mathrm{PT}$ method. The rotational entropy values obtained here show much less variation compared to the translational entropy with the variation of temperature. Also, minor change in the values at different temperatures for $\mathrm{TS}_{\mathrm{Rot}}$ is insignificant to be observed in Figure 3 (b).

Extrapolation of the translational entropy values above $240 \mathrm{~K}$ provides the temperature where translational entropy goes to zero. However, this is different from the Kauzmann temperature, $\mathrm{T}_{\mathrm{K}}$, which is obtained by extrapolating the configurational entropy values to zero. The Kauzmann temperature for SPC/E water has been reported to be $\sim 150 \mathrm{~K}$. The temperature at which the extrapolated translational entropy goes to zero is denoted here as $\mathrm{T}_{\text {Trans }}$. It is found to be $44.51 \mathrm{~K}$ for a single bulk water molecule. At this temperature, the water oxygen atoms will likely be static. This is shown in Figure 4(a). The slope of $\mathrm{TS}_{\text {Conf }} \mathrm{vs} \mathrm{T} / \mathrm{T}_{\mathrm{K}}$ gives the thermodynamic fragility of the system. Hence, we obtain an analog of this thermodynamic fragility, denoted here by $\mathrm{K}_{\text {Trans }}$, by obtaining the slope of the plot for $\mathrm{TS}_{\text {Trans }}$ vs $T / T_{\text {Trans }}$. This is shown in Figure 4(b). The value for $\mathrm{K}_{\text {Trans }}$ is found to be 0.02 .

\subsection{Diffusion coefficients (D) at different temperatures}

To obtain an insight on the dynamics of water molecules at different temperatures, we have calculated the selfdiffusion coefficient of water. To get the temperature dependence of $\mathrm{D}$, we have plotted the logarithm of the inverse of $\mathrm{D}$ with respect to temperature. This is shown in Figure 5. As seen from the figure, a non-Arrhenius behaviour appears for the $\mathrm{D}$ values at around $250 \mathrm{~K}$. A similar transition was observed by the quasi-elastic neutron scattering (QENS) experiment for the dynamics of water around the similar temperature range. ${ }^{47,48} \mathrm{How}-$ ever, in these studies, the transition at ambient pressure takes place at a slightly lower temperature compared to ours (around $225 \mathrm{~K}$ compared to $250 \mathrm{~K}$ in our case).

From the D, we can obtain the dynamic fragility of the system by fitting the non-Arrhenius segment using VFT equation given as Eq. 7. This is shown in Figure 5 by the solid line. Here we find that the $\mathrm{K}_{\mathrm{VFT}}$, known as the dynamic fragility marker to be 0.8 and $\mathrm{T}_{\mathrm{VFT}}$, the temperature at which glass transition takes place to be 204.8 K. These values are somewhat close to the ones reported by Chen et al. ${ }^{27}$ where the $\mathrm{T}_{\mathrm{VFT}}$ was found to be $187 \mathrm{~K}$ and $\mathrm{K}_{\mathrm{VFT}}$ is 0.56 . 


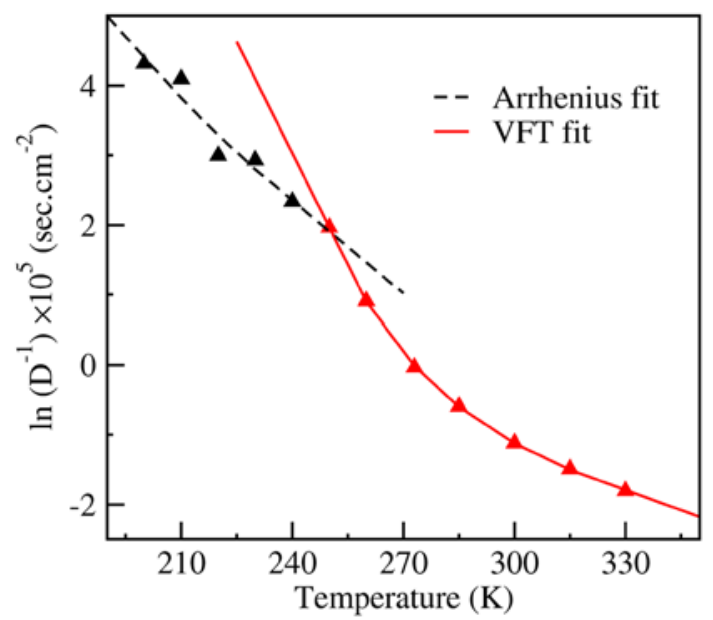

Figure 5. The logarithm of inverse diffusion coefficient with respect to temperature. The red solid line shows the VFT fit, while the black dashed line shows the Arrhenius fit.

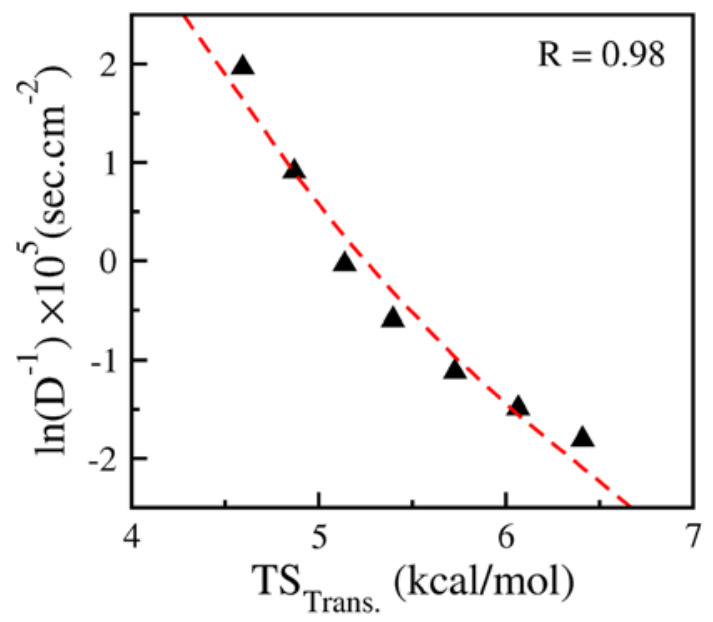

Figure 6. $\ln \left(\mathrm{D}^{-1}\right)$ is shown against $\mathrm{TS}_{\text {Trans }}$ for single water molecules. The red dashed line shows the fit with an analog of AG relation. $\mathrm{R}$ is the Pearson's correlation coefficient.

\subsection{Connecting the translational entropy with dynamics}

An analog of Adam-Gibbs (AG) relation has been used here to connect the entropy and diffusion coefficient. While the AG relation connects the configurational entropy with diffusion, we use the same form for connecting the translational entropy and diffusion by replacing the $\mathrm{TS}_{\text {Conf }}$ term in Eq. 3 by $\mathrm{TS}_{\text {Trans }}$. A similar approach has been used for connecting the tetrahedral entropy and tetrahedral relaxation time by Kumar et $a l .{ }^{50}$ The fit is shown in Figure 6. It can be seen that the fit for the translational entropy and diffusion with the AG relation is very good as indicated by the correlation coefficient. The $\mathrm{A}_{\mathrm{AG}}$ parameter, which indicates the high temperature barrier, is $60.6 \mathrm{kcal} / \mathrm{mol}$. Thus, our study indicates a relation between the diffusion and translational entropy for a single water molecule. To our knowledge, this is the first study that connects the translational entropy with the translational diffusion of water. The good fit also indicates that a common relation could be obtained between the translational entropy and diffusion so that from the knowledge of dynamics only (which is computationally cheap), the information on the thermodynamics (computationally costly and challenging) can be obtained. However to obtain a general behavior, the method will be needed to be applied on different systems.

\section{Conclusions}

In this study, we have calculated the translational and rotational entropy of single water molecules in the bulk at different temperatures. The values obtained here show the transition at a particular temperature. At the same temperature, diffusion coefficients show fragileto-strong transition. While kinetic fragility has been obtained from the diffusion coefficient, we used this translational entropy to calculate an equivalent description of thermodynamic fragility. Therefore, we have estimated the temperature at which the contribution of entropy originating from the translation of water vanishes. The thermodynamic and dynamic information follow an Adam-Gibbs type of relation. Therefore, this study shows that the present method can be used to obtain the computationally expensive and challenging quantity, entropy, from a faster and cheaper dynamical quantity, diffusion coefficient. Also, the new thermodynamic and kinetic fragility parameters provide new structural and dynamical information of a single water molecule in bulk water. Therefore, this method can be extended to study the dynamics and thermodynamics of water molecules in more complicated environments.

\section{Acknowledgements}

This work was funded by SERB DST, Government of India (EMR/2016/001069).

\section{References}

1. Goldstein M 1969 Viscous liquids and the glass transition: A potential energy barrier picture J. Chem. Phys. 513728

2. Scala A, Starr F W, La Nave E, Sciortino F and Stanley H E 2000 Configurational entropy and diffusivity of supercooled water Nature 406166 
3. Rosenfeld Y 1977 Comments on the transport coefficients of dense hard core systems Chem. Phys. Lett. 48 467

4. Rosenfeld Y 1977 Relation between the transport coefficients and the internal entropy of simple systems Phys. Rev. A 152545

5. ChakrabortyS N and Chakravarty C 2006 Diffusivity, excess entropy, and the potential-energy landscape of monatomic liquids J. Chem. Phys. 124014507

6. Yan Z, Buldyrev S V and Stanley H E 2008 Relation of water anomalies to the excess entropy Phys. Rev. E Stat. Nonlin. Soft Matter Phys. 78051201

7. Agarwal M, Singh M, Sharma R, Parvez Alam M and Chakravarty C 2010 Relationship between structure, entropy, and diffusivity in water and water-like liquids $J$. Phys. Chem. B 1146995

8. Agarwal M, Alam M P and Chakravarty C 2011 Thermodynamic, diffusional, and structural anomalies in rigid-body water models J. Phys. Chem. B 115 6935

9. Barros de Oliveira A, Salcedo E, Chakravarty C and Barbosa M C 2010 Entropy, diffusivity and the energy landscape of a water like fluid J. Chem. Phys. 132 234509

10. Nayar D and Chakravarty C 2013 Water and water-like liquids: Relationships between structure, entropy and mobility Phys. Chem. Chem. Phys. 1514162

11. Dhabal D, Nguyen A H, Singh M, Khatua P, Molinero V, Bandyopadhyay S and Chakravarty C 2015 Excess entropy and crystallization in Stillinger-Weber and Lennard-Jones fluids J. Chem. Phys. 143164512

12. Gallo P and Rovere M 2015 Relation between the twobody entropy and the relaxation time in supercooled water Phys. Rev. E: Stat. Nonlinear Soft Matter Phys. 91012107

13. Sengupta S, Vasisht V V and Sastry S 2014 Diffusivity anomaly in modified Stillinger-Weber liquids J. Chem. Phys. 140044503

14. Vasisht V V, Mathew J, Sengupta S and Sastry S 2014 Nesting of thermodynamic, structural, and dynamic anomalies in liquid silicon J. Chem. Phys. 141124501

15. Baranyai A and Evans D J 1989 Direct entropy calculation from computer simulation of liquids Phys. Rev. A 403817

16. Dzugutov M 1996 A universal scaling law for atomic diffusion in condensed matter Nature 381137

17. Seki K and Bagchi B 2015 Relationship between entropy and diffusion: A statistical mechanical derivation of Rosenfeld expression for a rugged energy landscape $J$. Chem. Phys. 143194110

18. Adam G and Gibbs J H 1965 On the temperature dependence of cooperative relaxation properties in glassforming liquids J. Chem. Phys. 43139

19. Sastry S 2001 The relationship between fragility, configurational entropy and the potential energy landscape of glass-forming liquids Nature 409164

20. Sengupta S, Vasconcelos F, Affouard F and Sastry S 2011 Dependence of the fragility of a glass former on the softness of interparticle interactions J. Chem. Phys. 135194503

21. Banerjee A, Sengupta S, Sastry S and Bhattacharyya S M 2014 Role of structure and entropy in determining differences in dynamics for glass formers with different interaction potentials Phys. Rev. Lett. 113225701

22. Banerjee A, Nandi M K, Sastry S and Bhattacharyya S M 2016 Effect of total and pair configurational entropy in determining dynamics of supercooled liquids over a range of densities J. Chem. Phys. 145034502

23. Nandi M K, Banerjee A, Sengupta S, Sastry S and Bhattacharyya S M 2015 Unraveling the success and failure of mode coupling theory from consideration of entropy J. Chem. Phys. 143174504

24. Angell C A, 1982 Water: A comprehensive treatise (New York: Springer)

25. Bergman R and Swenson J 2000 Dynamics of supercooled water in confined geometry Nature $\mathbf{4 0 3} 283$

26. Koga K, Tanaka H and Zeng X C 2000 First-order transition in confined water between high-density liquid and low-density amorphous phases Nature 408564

27. Chen S H, Mallamace F, Mou C Y, Broccio M, Corsaro C, Faraone A and Liu L 2006 The violation of the StokesEinstein relation in supercooled water Proc. Natl. Acad. Sci. USA 10312974

28. Sasikala W D and Mukherjee A 2014 Single water entropy: Hydrophobic crossover and application to drug binding J. Phys. Chem. B 11810553

29. Siebert X and Amzel L M 2004 Loss of translational entropy in molecular associations Proteins: Struct. Funct. Bioinf. 54104

30. Angell A 1998 Thermodynamics: Liquid landscape Nature 393521

31. Ito K, Moynihan C T and Angell C A 1999 Thermodynamic determination of fragility in liquids and a fragile-to-strong liquid transition in water Nature 398 492

32. Rick S W 2004 A reoptimization of the five-site water potential (TIP5P) for use with Ewald sums J. Chem. Phys. 1206085

33. Hess B, Kutzner C, van der Spoel D and Lindahl E 2008 GROMACS 4: Algorithms for highly efficient, loadbalanced, and scalable molecular simulation J. Chem. Theory Comput. 4435

34. Schlick T 2010 Molecular modelling and simulation: An interdisplinary guide (New York: Springer).

35. Berendsen H J C, Postma J P M, Vangunsteren W F, Dinola A and Haak J R 1984 Molecular-dynamics with coupling to an external bath J. Chem. Phys. 81 3684

36. Nose S 1984 A molecular-dynamics method for simulations in the canonical ensemble Mol. Phys. 52255

37. Hoover W G 1985 Canonical dynamics-Equilibrium phase-space distributions Phys. Rev. A 311695

38. Parrinello M and Rahman A 1981 Polymorphic transitions in single-crystals-A new molecular-dynamics method J. Appl. Phys. 527182

39. Darden T, York D and Pedersen L 1993 Particle mesh Ewald: An N.log(N) method for Ewald sums in large systems J. Chem. Phys. 9810089

40. Mallamace F, Branca C, Broccio M, Corsaro C, Mou C Y and Chen S H 2007 The anomalous behavior of the density of water in the range $30 \mathrm{~K}<\mathrm{T}<373 \mathrm{~K}$ Proc. Natl. Acad. Sci. USA 10418387

41. McQuarrie D A 2000 Statistical mechanics (New York: University Science Books) 
42. Karplus M and Kushick J N 1981 Method for estimating the configurational entropy of macromolecules Macromolecules 14325

43. Reinhard F and Grubmüller H 2007 Estimation of absolute solvent and solvation shell entropies via permutation reduction J. Chem. Phys. 126014102

44. Reinhard F, Lange O F, Hub J S, Haas J and Grubmüller H 2009 g_permute: Permutation-reduced phase space density compaction Comput. Phys. Commun. 180455

45. Andricioaei I and Karplus M 2001 On the calculation of entropy from covariance matrices of the atomic fluctuations J. Chem. Phys. 1156289

46. Saha D and Mukherjee A 2016 Impact of ions on individual water entropy J. Phys. Chem. B 1207471
47. Faraone A, Liu L, Mou C Y, Yen C W and Chen S H 2004 Fragile-to-strong liquid transition in deeply supercooled confined water J. Chem. Phys. 12110843

48. Liu L, Chen S H, Faraone A, Yen C W and Mou C Y 2005 Pressure dependence of fragile-to-strong transition and a possible second critical point in supercooled confined water Phys. Rev. Lett. 95117802

49. Lin S T, Maiti P K and Goddard W A 2010 Two-phase thermodynamic model for efficient and accurate absolute entropy of water from molecular dynamics simulations J. Phys. Chem. B 1148191

50. Kumar P, Buldyrev S V and Stanley H E 2009 A tetrahedral entropy for water Proc. Natl. Acad. Sci. USA 106 22130 